\title{
Effect of Chemical Treatment on the Tensile Properties of Single Oil Palm Empty Fruit Bunch (OPEFB) Fibre
}

\author{
Hassan CS ${ }^{1 *}$, Sapuan $\mathrm{SM}^{2}$, Abd AzizN²and Mohamed Yusof $\mathrm{MZ}^{2}$ \\ ${ }^{1}$ Department of Mechanical Engineering, UCSI University, Malaysia \\ ${ }^{2}$ Department of Mechanical and Manufacturing Engineering, University Putra Malaysia, Malaysia \\ *Corresponding author: Hassan CS, Mechanical Engineering Department, UCSI University, Jalan Menara Gading, UCSI Heights, 56000 Cheras, Kuala \\ Lumpur, Malaysia
}

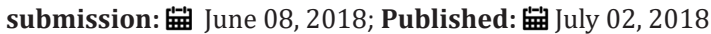

\begin{abstract}
A study on effect of chemical treatment on the tensile modulus and strength of single oil palm empty fruit bunch (OPEFB) fibre is presented in this paper. The fibres were treated with alkaline treatment using sodium hydroxide of $5 \%$ concentration. Tensile test were conducted in accordance to the ASTM C1557. Results revealed that the tensile modulus of the treated fibres decreases by around $55 \%$ as compared to the untreated fibres. Tensile strength on the other hand increases by around $25 \%$ with the alkaline treatment. The tensile strength was found to be dependent on the fibre diameter where the strength decreases with the increases in fiber diameter at fracture. SEM revealed higher porosity at larger fibre diameter which contributed to the degradation of the fibre tensile strength. No significant impact by the alkaline treatment was observed to the tensile strain of the fibre.
\end{abstract}

Keywords:OPEFB fibre; Natural fibre; Tensile strength OPEFB fibre; Tensile modulus OPEFB fibre

\section{Introduction}

As one of the largest palm oil producer, Malaysia also generates a large amount of oil palm biomass. Abdullah \& Sulaiman [1] stated that there is abundance of raw materials available from the palm tree consisting of around $90 \%$, by weight, of non-fruit biomass. Oil palm biomass can be derived from three sources, that is, oil palm frond, fruit bunch and oil palm trunk, as depicted in Figure 1.

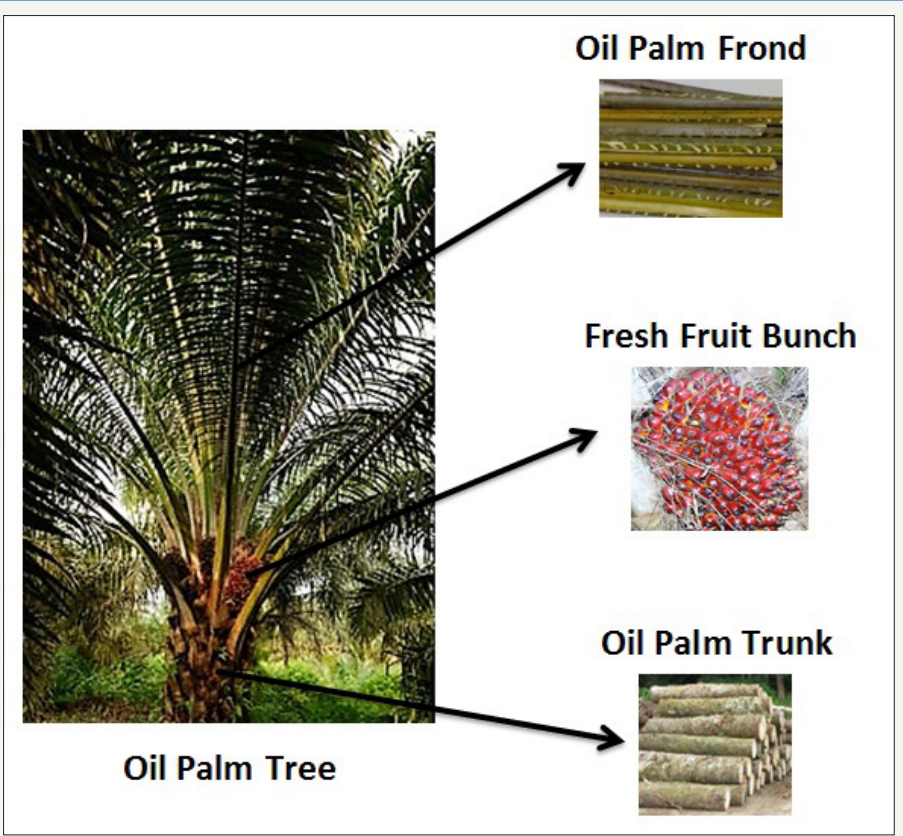

Figure 1: Structure of oil palm tree. 
Fibres from oil palm frond and trunk are obtained directly from the plantation site, whereas fibres from fresh fruit bunch are obtained from palm mill site. The process of extracting crude palm oil from the fresh fruit bunches in turns generates a large amount of biomass residue. Among the biomass generated is OPEFB fibre. It is extracted by retting process from the empty fruit bunch [2]. It is a fibrous mass left behind after separating the fruits from the fresh fruit bunches [3].

OPEFB fibre is one of the most targeted oil palm biomass residues in the market which could be utilized to produce polymer bio-composite, pulp, biofuel and many other products. In order to effectively utilize OPEFB fibre as polymer bio-composite, extensive researches have been carried out over the years to determine the applicability of the OPEFB fibre to be incorporated into the abovementioned applications. One of the drawbacks in the fabrication of the fibre reinforced composite is the ability to attain good bonding between the fibre and the matrix. Chemical treatment such as alkaline treatment is the most commonly utilized method to promote better bonding between the fibre and the matrix and therefore improve the mechanical properties of the composite. Chemical treatment resulted in the removal of lignin, wax and oils covering the external surface of the fibre wall thereby increasing the surface roughness of the fibre which is needed to achieve good bonding with matrix.

The effects of chemical treatment on the structural, thermal, physical, mechanical and morphological properties of roselle fibre reinforced vinyl ester composites were studied by Nadlene [4].
Alkalization treatment has found to improve the thermal stability of the roselle fibre due to the removal of lignin and hemicellulose in the fibre structure, which exposes more cellulose. Tensile strength was found to improve but impact properties on the other hand decreases. Norizan [5] studied the effect of silane treatment on the mechanical and thermal properties of sugar palm fibre reinforced polyurethane composites. The silane treatment has been shown to contribute to an enhancement in mechanical properties, including tensile, flexural and impact properties of the composites. Hassan [6] investigated the effect of chemical treatment on the water absorption and tensile properties of OPEFB fibre reinforced epoxy composite. Treated fibre shown to be able to increase the tensile strength of the composite.

While the treatment has been proven to be able to improve bonding and strength of composite, the effect of the treatment to the fibre itself has not yet been investigated. Our aim is to analyze the effect of chemical treatment to the tensile properties of single OPEFB fibre.

\section{Materials and Methods}

The fibre used is in tangle form, as shown in Figure 2. It was dismantled to form individual fibre as shown in Figure 3. After dismantling of OPEFB fibre, the fibres were washed with distilled water for about 15-20 times until the water did not change colour. It was washed in order to remove the impurities such as residue oil and other large particles. The fibres then were allowed to dry in natural ventilation for 24 hours at room temperature. Some of the fibre were separated from here and labelled as untreated fibre.

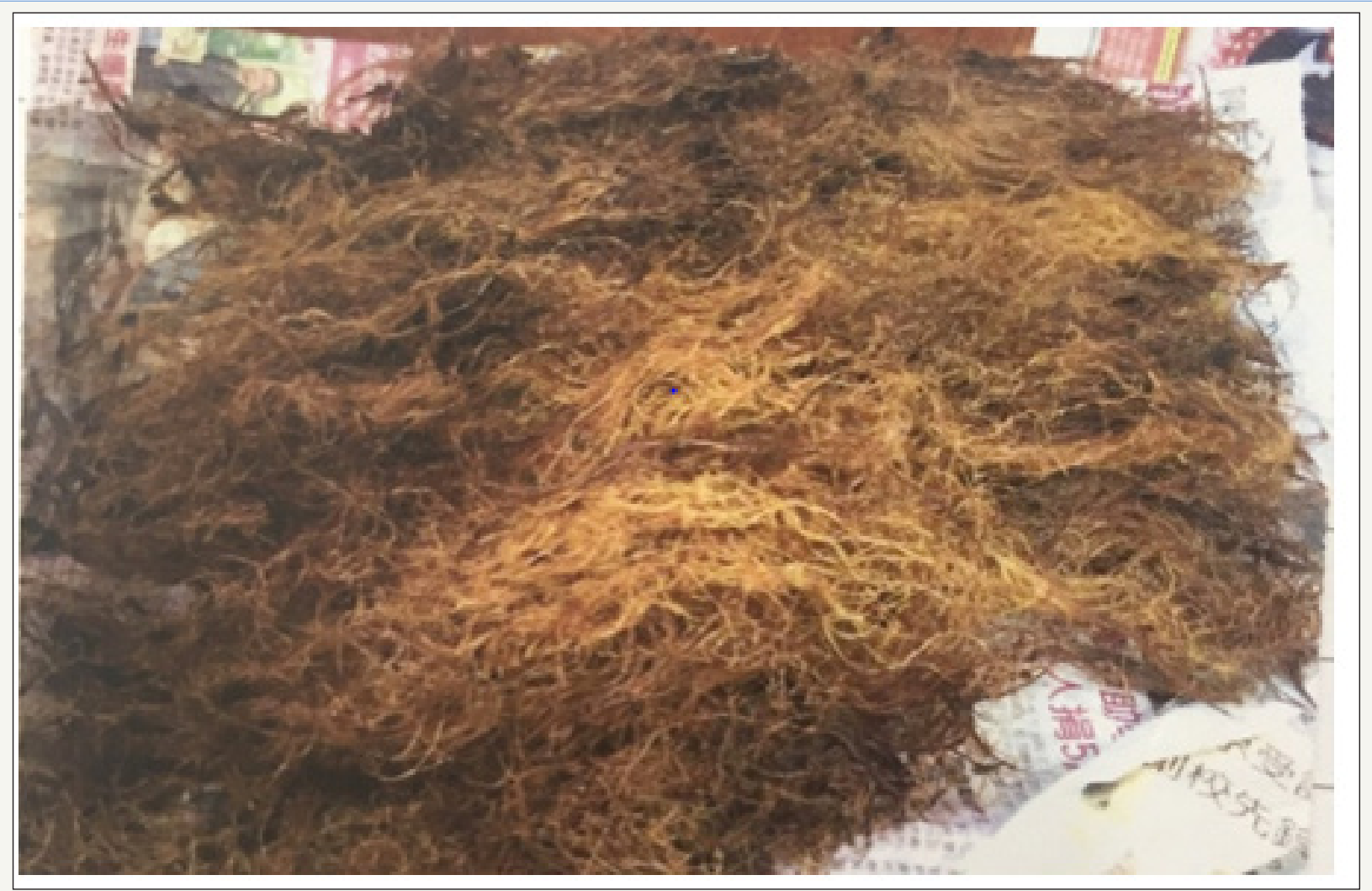

Figure 2: Bundle OPEFB fibres. 


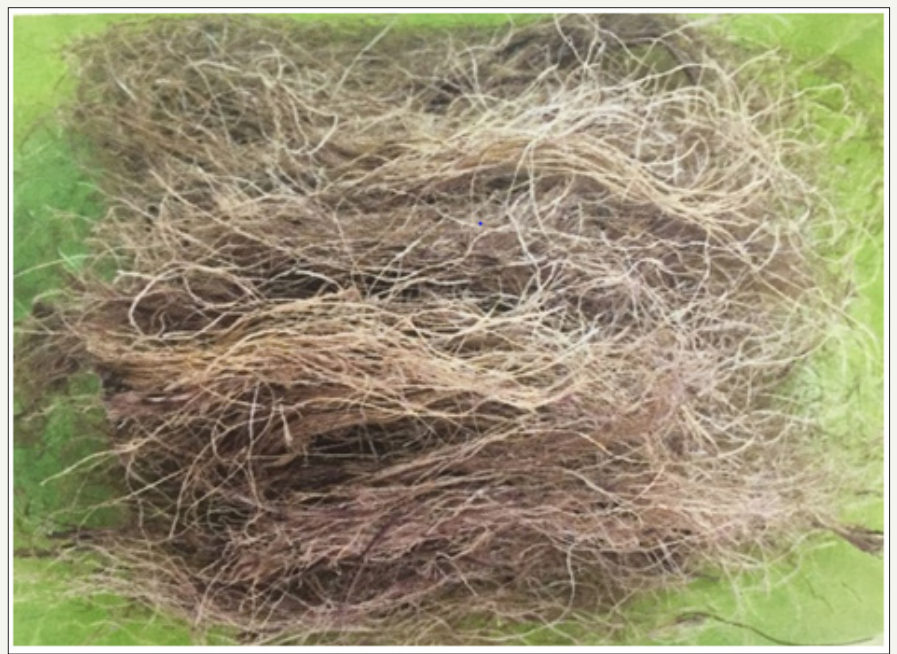

Figure 3: Dismantled OPEFB fibre.

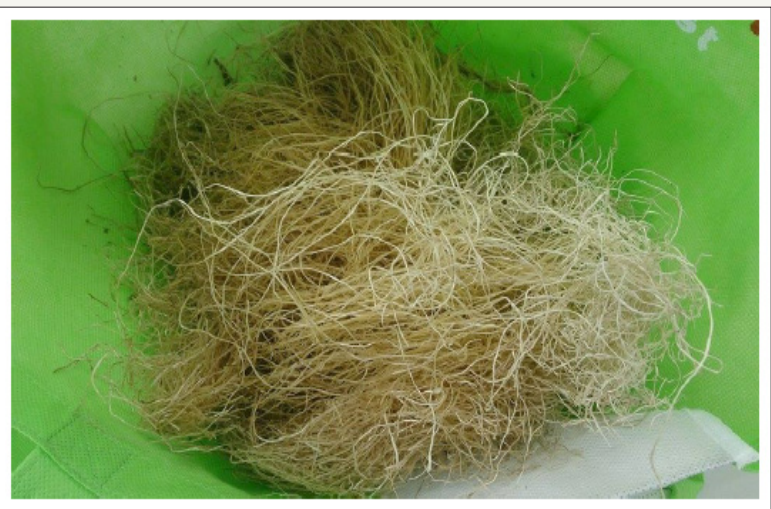

Figure 4: Treated OPEFB fibres.

The remaining fibres were then soaked in a $5 \%$ sodium hydroxide solution for 1 hour. The fibres were then washed again with distilled water and at every interval of 2 washes; a litmus paper was dipped into the distilled water which contained the fibres to test for the presence of alkali. Once the alkali has been removed completely, the fibres were allowed to dry for another 24 hours at room temperature. This fibre is labelled as alkaline treated fibre, shown in Figure 4.

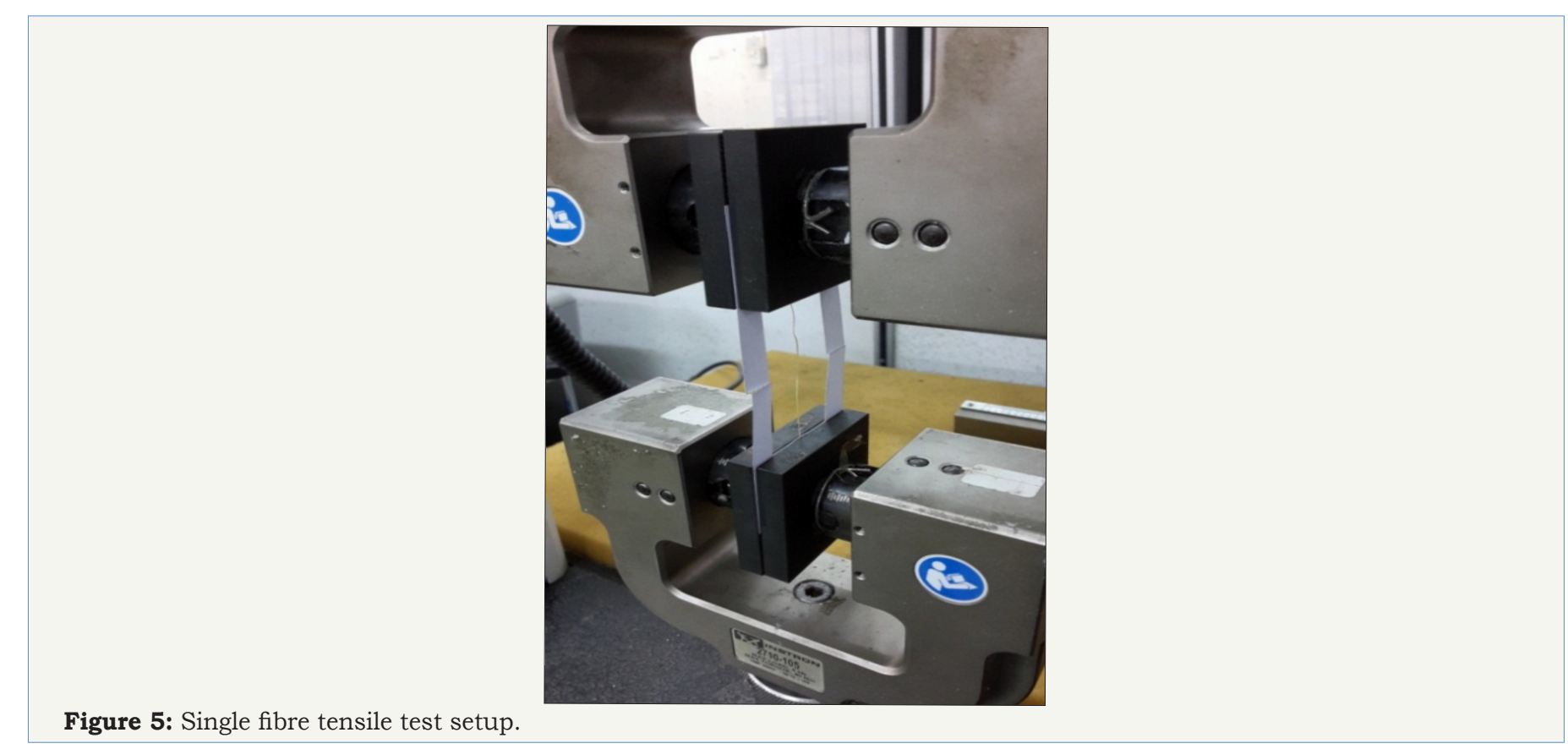


Single fibre strand tension test is conducted to determine the stress to strain behaviour of the OPEFB fibre. Tensile modulus and tensile strength are obtained from the test stress strain curve. Tensile test of the single OPEFB fibre were conducted in accordance to the ASTM C1557. Single filament was fixed at both ends of the slot in the paper tab using adhesive. Fibre gauge length of $50 \mathrm{~mm}$ was used for the entire test. Displacement as a function of the applied load was recorded during the entire test until the fibre break. The test was conducted using a 5kN INSTRON 3365 machine with a cross-head speed of $2 \mathrm{~mm} / \mathrm{min}$. Both sides of the tab were cut at the mid-gage prior to testing. A typical test sample and setup is shown in Figure 5. Tensile strength for the single fibre was calculated using equation 1.

$$
\sigma=\frac{F}{A}
$$

Where $\sigma$ is the tensile strength, $F$ is the highest value of force achieved and $A$ represents the fiber cross-sectional area at fracture plane. The cross sectional diameter of the fractured areas was measured using a microscope.

\section{Results and Discussion}

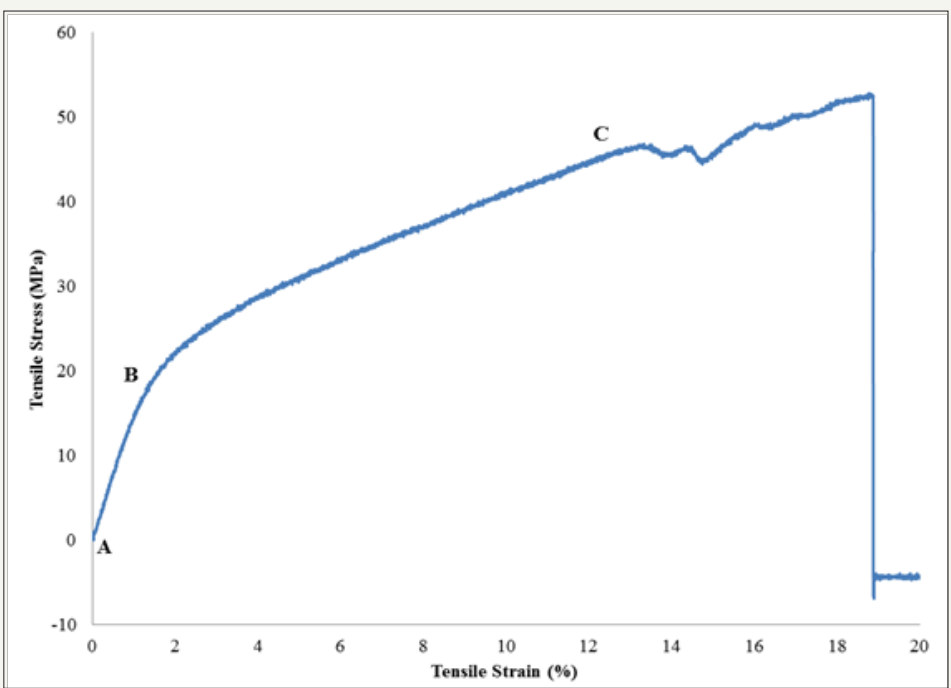

Figure 6: Tensile stress-strain curve of single OPEFB fibre.

The experiment was conducted on two type of specimen that is untreated OPEFB fibres and $\mathrm{NaOH}$ treated OPEFB fibres. Typical stress-strain curve of the OPEFB fibre obtained in the single fibre tensile test is shown in Figure 6. The stress-strain curve shows the relationship between the magnitude of the applied stress on a material and the resulting strain. The curve exhibited elastic deformation region $(\mathrm{AB})$ until strain around $1.25 \%$, followed by plastic deformation and failure.

Figure 7: Untreated OPEFB fibre surface.

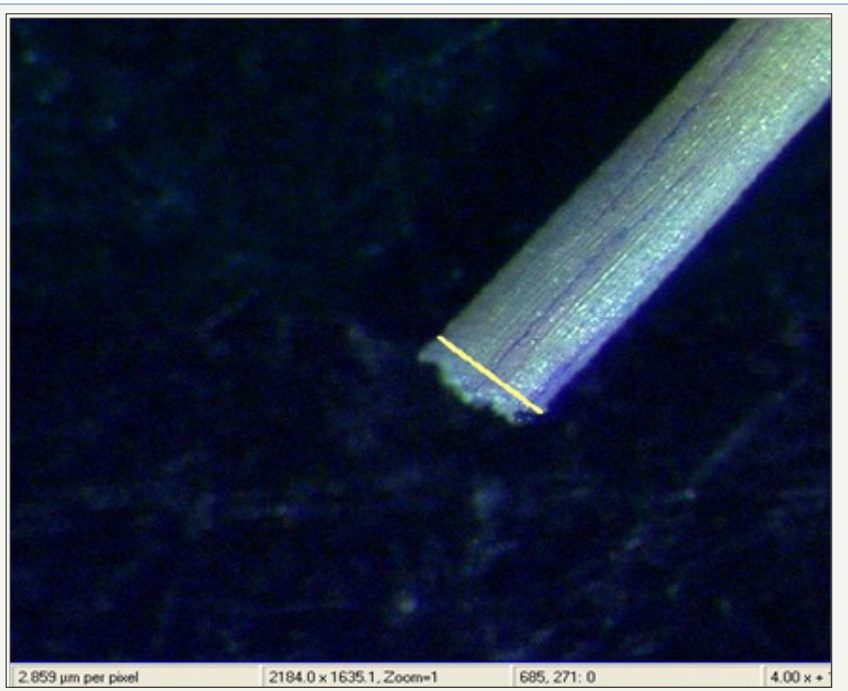

The fibre modulus was evaluated from the slope of the curve in the elastic deformation region. Tensile modulus of the single untreated OPEFB fibre was found to be on average of $1325.63 \mathrm{MPa}$. The tensile modulus of single $\mathrm{NaOH}$ treated OPEFB fiber meanwhile found to be around $595.88 \mathrm{MPa}$. The modulus of elasticity of the OPEFB fibre was found to decrease of around 55\% after alkaline 
treatment. Similar findings have been reported by Osorio et al. [7] and Arifuzzaman et al. [8] for banana and oakra fibre, respectively. The decreases in tensile modulus after alkaline treatment may have been due to cellulose delignification and degradation during alkali treatment which disrupting bonding and leading to morphological changes, like increased surface roughness. Figure $7 \& 8$ shows the fibre surface of the untreated and treated OPEFB fibre, respectively. Comparing Figure $7 \& 8$, it can be seen that the surface of the OPEFB fibre became rougher upon subjected to alkaline treatment. Such increased roughness acts as stress concentrator which weakened the fibres' outer wall thereby decreasing the rigidity of the fibre.
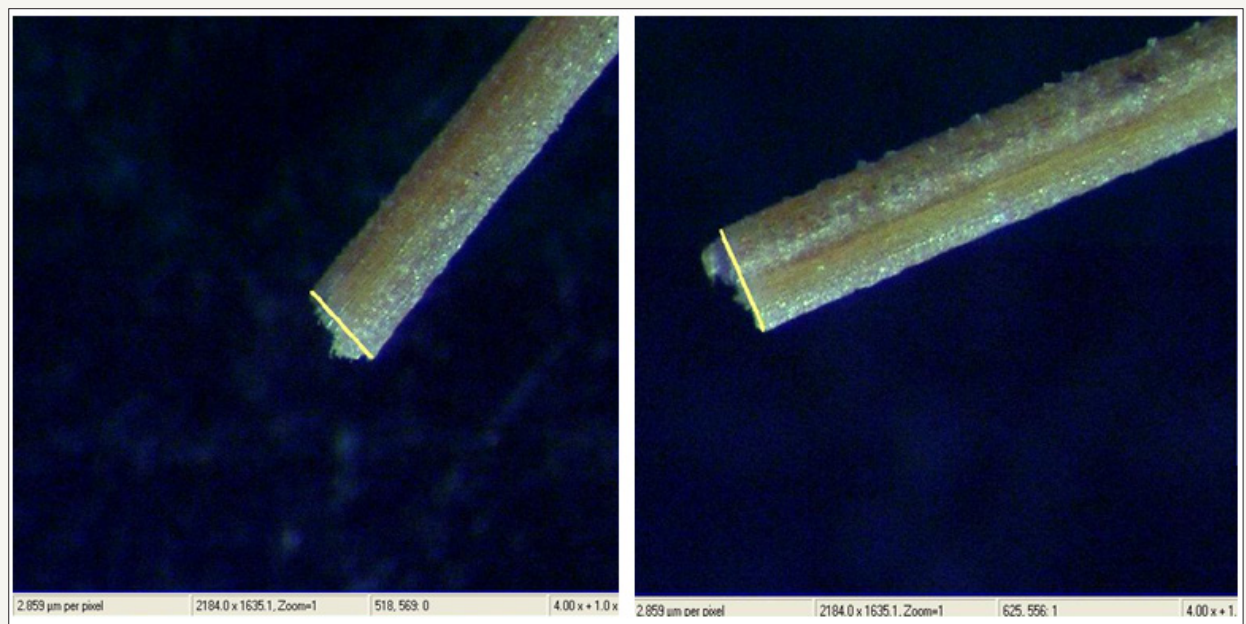

Figure 8: $\mathrm{NaOH}$ treated OPEFB fibre surface.

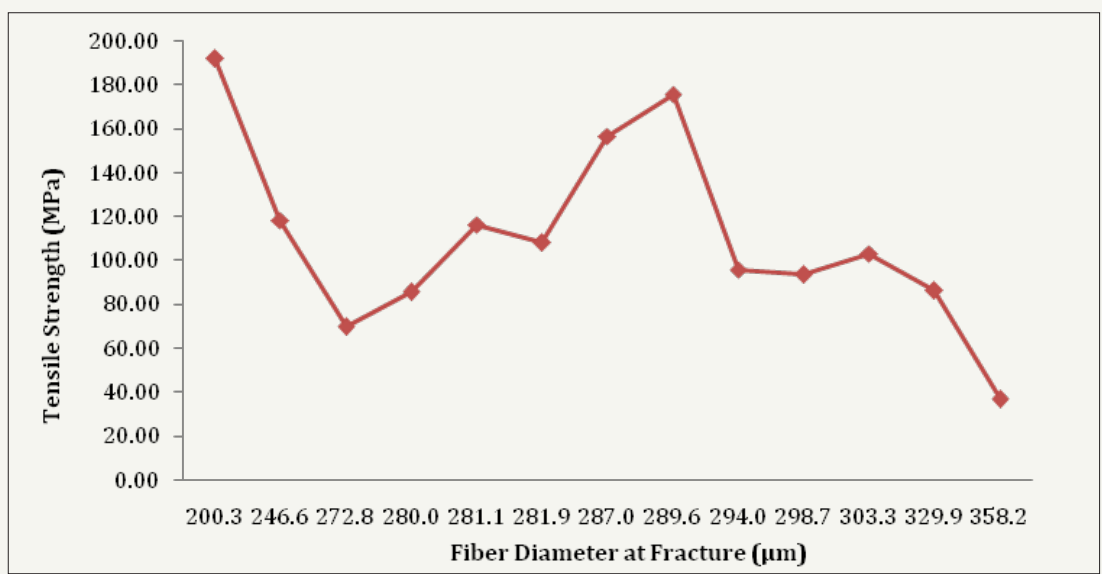

Figure 9: Tensile strength of single untreated OPEFB fiber.

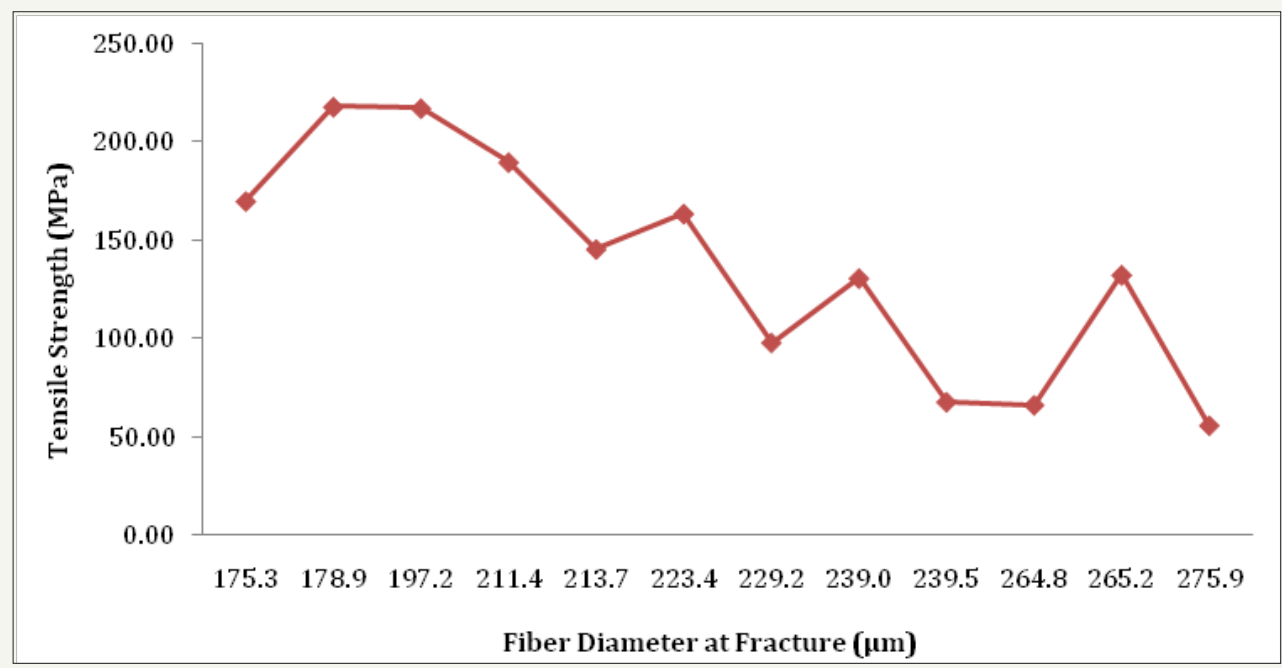

Figure 10: Tensile strength of single $\mathrm{NaOH}$ treated OPEFB fiber. 
Figure $9 \& 10$ shows the tensile strength of the untreated and $\mathrm{NaOH}$ treated OPEFB fibre, respectively. The tensile strength was found to be dependent on the fibre diameter where the strength decreases with the increases in fiber diameter at fracture. The average fibre diameter for the untreated OPEFB fibre was $366 \mu \mathrm{m}$ while the average fibre diameter at fracture for the $\mathrm{NaOH}$ treated fibre was $363 \mu \mathrm{m}$. The degradation of tensile strength at higher fibre diameter might be due to the imperfection found in each fiber. The imperfection in natural fibre exists in the form of fibre porosity or lumen cavities. These imperfections were seen through SEM analysis shown in Figure $11 \& 12$. Fibre with higher diameter and length is believed to contain higher porosity thus reducing the tensile properties of fiber. The presence of the porosity modified the real cross-section of the fibre and therefore the mechanical properties. However, inconsistencies were indeed observed at a few fiber diameters. A few fibers were found to have higher tensile strength value even though fracture was observed at higher fiber diameter. This might be attributed to lower porosity content of those fibers although at higher fiber diameter. Tensile strength for the single untreated OPEFB fibre was found between $36 \mathrm{MPa}$ to $192 \mathrm{MPa}$, with an average of $110.43 \mathrm{MPa}$. Meanwhile, tensile strength for the alkaline treated single OPEFB fibre was found to be in the range of $57 \mathrm{MPa}$ to $218 \mathrm{MPa}$, with an average of $137.87 \mathrm{MPa}$.

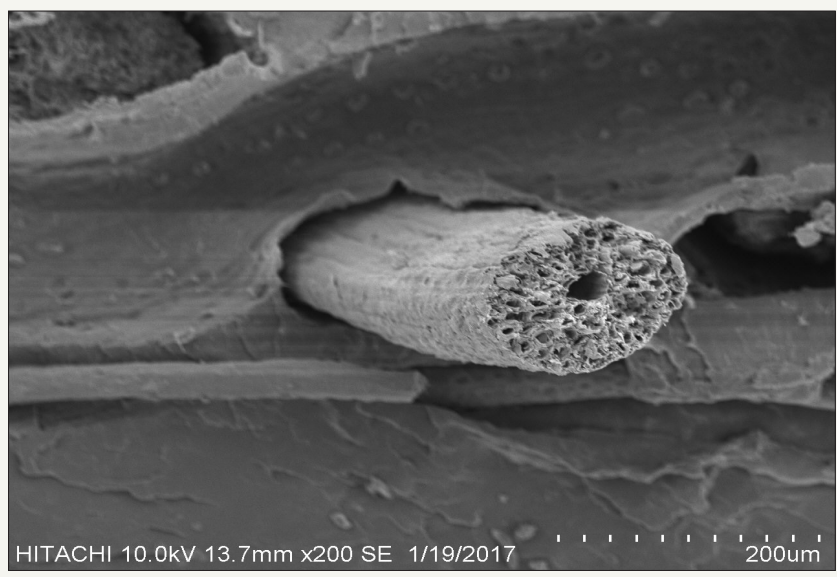

Figure 11: Angular view of OPEFB fibre surface with high presence of porosity.

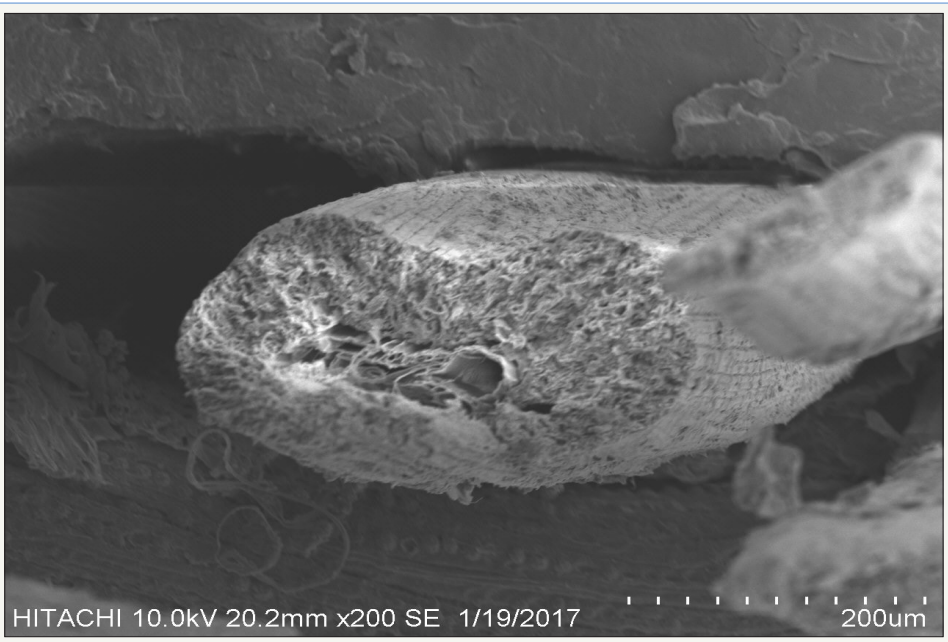

Figure 12: Angular view of OPEFB fibre surface with low presence of porosity.

Despite the decreases in tensile modulus after alkaline treatment, the tensile strength is observed to increase. Due to the removal of hemicellulose and lignin by chemical treatment which made the fibre to be less rigid, the fibrils would be able to rearrange themselves along the direction of the tensile load applied. The smoother rearrangement of the fibrils would result in better load sharing hence increases the tensile strength of the fibre [9].

Mohamed [10] reported single untreated OPEFB fibre with tensile strength, Young's modulus and elongation at break of 71MPa,
$1703 \mathrm{MPa}$ and 11, respectively. Diameters of the fibres tested were in the range of 250 to $610 \mu \mathrm{m}$. Value variation between the author result and present study can be explained by defects such as crossmarks, kink bands or dislocations that become stress concentrators [11], the fibre's internal structure causing a non-constant crosssection along fibre length and variability in fibre composition [7] and the difference in diameter of fibre tested.

No significant impact by the alkaline treatment was observed to the tensile strain of the fibre. Tensile strain of the untreated OPEFB 
fibre was found to be in the range of $14 \%$ to $42 \%$ with an average of $26.85 \%$ while the tensile strain of the $\mathrm{NaOH}$ treated OPEFB fibre was in between $15 \%$ to $33 \%$ with an average of $26.11 \%$.
The tensile properties of the single OPEFB fibre are summarized through Figure 13.

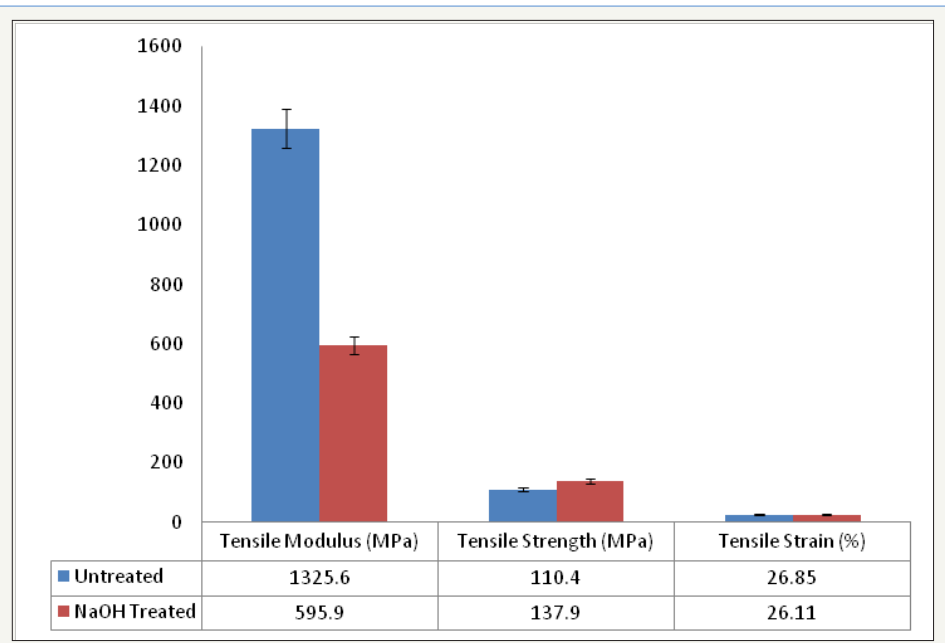

Figure 13: Tensile property of single OPEFB fibre.

\section{Conclusion}

Tensile properties of the single OPEFB fibre subjected to alkaline treatment were investigated. The OPEFB fibre exhibited stress-strain curve with linear elastic region which subsequently followed by yielding, plastic deformation and failure. The modulus of elasticity of the alkaline treated OPEFB fibre was found to decrease by around $55 \%$ as compared to the non-treated OPEFB fibre. The tensile strength on the other hand increases by around $25 \%$. No significant changes were observed to the tensile strain of the fibre with the alkaline treatment.

\section{References}

1. Abdullah N, Sulaiman F (2013) The oil palm wastes in Malaysia. Biomass Now-Sustainable Growth and Use, pp. 75-100.

2. Hassan A, Salema AA, Ani FN, \& Bakar AA (2010) A review on oil palm empty fruit bunch fibre-reinforced polymer composite materials. Polymer Composites 31(12): 2079-2101.

3. Faruk O, Sain M (2014) Biofiber reinforcements in composite materials. Elsevier Science, USA.

4. Nadlene R, Salit MS, Jawaid M, Ishak MR, Yusriah L (2018) The effects of chemical treatment on the structural and thermal, physical, and mechanical and morphological properties of roselle fiber-reinforced vinyl ester composites. Polymer Composites 39(1): 274-287.
5. Norizan MN, Abdan K, Salit MS, Mohamed R (2018) The effect of alkaline treatment on the mechanical properties of treated sugar palm yarn fibre reinforced unsaturated polyester composites reinforced with different fibre loadings. Sains Malaysiana 47(4): 699-705.

6. Hassan CS, Chellaiah NR, Sahari B, Salit MS, Abdul AN (2016) Effect of chemical treatment on oil palm empty fruit bunch (OPEFB) fiber on water absorption and tensile properties of opefb fiber reinforced epoxy composite. Key Engineering Materials 701: 295-299.

7. Osorio JCM, Baracaldo RR, Florez JJO (2012) The influence of alkali treatment on banana fibre's mechanical properties. Engineering and Research 32(1): 83-87.

8. Arifuzzaman KGM, Shaheruzzaman M, Rahman MH, Abdur Razzaque SM, Islam MS, et al. (2009) Surface modification of okra bast fiber and its physico-chemical characteristics. Fibers and Polymers 10(1): 65-70.

9. Bledzki AKG (1999) Composites reinforced with cellulose based fibers. Progress in Polymer Science 24(2): 221-274.

10. Mohamed YMZ, Salit MS, Ismail N (2009) Tensile properties of single oil palm empty fruit bunch (OPEFB) fibre. Sains Malaysiana 38(4): 525-529.

11. Baley C (2002) Analysis of the flax fibres tensile behaviour and analysis of the tensile stiffness increase. Composites Part A: Applied Science and Manufacturing 33(7): 939-948.
Creative Commons Attribution 4.0 International License

For possible submissions Click Here

\section{Submit Article}

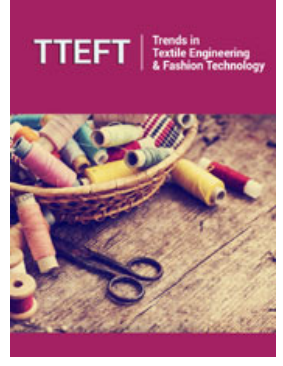

Trends in Textile Engineering \& Fashion Technology

\section{Benefits of Publishing with us}

- High-level peer review and editorial services

- Freely accessible online immediately upon publication

- Authors retain the copyright to their work

- Licensing it under a Creative Commons license

- Visibility through different online platforms 